\title{
Implementation and Findings On A One-Minute Sit- Stand Test for Prehospital Triage in Patients With Suspected COVID-19 - A Pilot Project
}

Julie Linding Kjerulff ( $\nabla$ julkje@rm.dk)

Central Denmark Region

\section{Allan Bach}

Central Denmark Region

Ulla Væggemose

Central Denmark Region

Søren Helbo Skaarup

Aarhus University Hospital, Central Denmark Region

Morten Thingemann Bøtker

Central Denmark Region

\section{Research Article}

Keywords: Prehospital Emergency Medical Services, Exercise testing, Corona virus disease 2019

Posted Date: October 5th, 2021

DOl: https://doi.org/10.21203/rs.3.rs-914952/v1

License: (9) This work is licensed under a Creative Commons Attribution 4.0 International License.

Read Full License

Version of Record: A version of this preprint was published at BMC Emergency Medicine on March 31st, 2022. See the published version at https://doi.org/10.1186/s12873-022-00605-9. 


\section{Abstract}

\section{Introduction:}

During the initial Coronavirus Disease 2019 (COVID-19) pandemic wave, sparse personal protection equipment made telephone triage of suscpeted COVID-19 patients for ambulance transport necessary. To spare resources, stable patients were often treated and released on-scene, but reports from Italy suggested that some later detoriated. We implemented a prehospital sit-stand test to identify patients in risk for detoriation.

\section{Methods}

The test was implemented as part of a new guideline in stable suspected COVID-19 patients younger than 70 years with no risk factors for serious disease triaged by general practitioners to ambulance response in the Central Denmark Region. Data were collected from April 6th to July 6th 2020. This was a two-part study evaluating guideline adherence and results of the sit-stand test. The primary outcome of each separate part was 1) the proportion of patients sit-stand tested before treat-and-released and 2) the proportion of patients treated with oxygen within 7 days among patients decompensating and not decompensation during the test.

\section{Results}

Data on 156 patients triaged to ambulance response by general practioners were analysed. In total $86 / 156$ (55\%) were tested with the sit-stand test, among these 30/86 (47\%) were either older than 70 or had risk factors for serious disease. Of those treated and released, 50/52 (96\%) were tested. In total, $17 / 86(20 \%)$ decompensated during the test and of these, $9 / 17$ (53\%) were treated with oxygen compared to $2 / 69(3 \%)$ in patients who did not decompensate $(p<0.001)$. This difference was only significant in the elderly group of patients with risk factors for serious disease. 10/156 (6\%) of patients had a positive COVID-19-test.

\section{Conclusion}

The sit-stand test was implemented in $96 \%$ of patients treated and released on-scene, but was also used in elderly patients with risk factors for serious disease. Decompensation was observed in $20 \%$ of patients and was associated with oxygen treatment within 7 days - but only in the elderly group of patients with risk factors for serious disease. These findings are hypotheses-generating and suggest that physical exercise testing may be usefull for decision making in emergency settings.

\section{Introduction}


Coronavirus Disease 2019 (COVID-19) rapidly turned into a worldwide pandemic [1, 2] and caused massive resource consumption that in some regions exceeded the maximal capacity of the health care system $[3,4]$.

COVID-19 disease can lead to acute lung injury, which necessitates hospital admission and oxygen therapy, according to initial reports in approximately $20 \%$ of patients $[7,8]$. The majority of patients, approximately $80 \%$, have a mild presentation and do not require neither hospital admission nor supplemental oxygen $[5,6]$. Due to sparse personal protective equipment in Denmark during the initial pandemic wave, general practioners (GPs) were prevented from physical examination of patients and telephonic assessment of suspected COVID-19 patients became necessary. Decision-making on whether to triage for ambulance transport was in this period thus based merely on a telephone interview of the patient or next-of-kin. On ambulance arrival, a large proportion of patients presented with stable vital signs and in order to reserve hospital and ambulance resources, stable patients without risk factors for serious disease were often treated and released on-scene. However, reports spreaded from Italy that seemingly stable COVID-19 patients not initially admitted to hospital later developed severe respiratory failure - a feature caused by cytokine storm phase $[7,8]$. It was also reported that physical exercise testing could be used to identify the group of seemingly stable COVID-19 patients, who would later deteriorate.

The one-minute sit-stand test is a functional exercise capacity test used to evaluate physiologic reserves used in patients with lung disease [9-12]. It correlates well with the validated six-minute walk test, but contrary to the six-minute test, it is feasible in a prehospital setting $[11,13]$. We implemented the sit-stand test as part of a clinical guideline for management of suspected COVID-19 patients in ambulances in the Central Denmark as a precautionary measure before treating and releasing seemingly stable low-risk patients on-scene. The aim of this study was to evaluate guideline adherence on the use of the prehospital one-minute sit-stand test and to examine, if decompensation during the test was in fact useful for identification of seemingly stable patients suspected of COVID-19, who would later develop a need for oxygen treatment.

\section{Materials And Methods}

The study was a quality assurance project with prospectively collected data on the implementation of and results of a sit-stand test on patients with suspected COVID-19 receiving an ambulance in the Central Denmark Region during the intial pandemic wave (from 6th April 2020 to 6th July 2020). According to Danish ethical and legal regulations, informed consent from the patients was not required for datacollection for this quality assurance project (this waiver was approved by the Central Denmark Region Committees on Health Research Ethics).

\section{Setting and implementation}

Central Denmark Region covers an area of $13,053 \mathrm{~km}^{2}$ and 1.3 million inhabitants corresponding to $23 \%$ of the Danish population [14]. Patients were triaged for ambulance transport either by GP via telephone 
interview, visit in GP clinic or GP home visit or following a citizen call to the emergency 1-1-2 number where medical personnel at the emergency medical communication center (EMCC) undertook criteriabased triage [15].

According to guidelines implemented before the COVID-19 pandemic, patients who had been triaged for ambulance transport after physical examination by a GP were not to be treated and released on-scene by ambulance personnel, whereas patients triaged after a telephone consultation only (GP or 1-1-2 call) could be treated and released after consultation with a physician [16].

During the initial pandemic wave, when GP's were prevented from physically examining suspected COVID19 patients due to lack of personal protective equipment, a new guideline for prehospital management of telephone triaged patients was implemented. According to this guideline, patients with known risk factors for progression to serious disease (i.e. age $>70$, significant comorbidities and/or unstable vital parameters) were to be taken to hospital for further evaluation [17]. In stable patients $<70$ years of age with no risk factors for serious disease, the one-minute sit-stand test was implemented as precautionary security measure before considering treating and releasing the patient on-scene (English translation of decision-support flow-chart in Fig. 1). Implementation of this new guideline was conducted according to organizational routines, as a written guideline distributed throughout the organisation. The guideline was supplemented by an instructional video describing which patients should be tested and how to perform the one-minute sit-stand test. Patients could at any time deny participation in exercise test.

\section{Exercise testing and clinical decision-making}

The one-minute sit-stand test was conducted by the ambulance personnel on-scene according to Fig. 1 . Prior to the test, all patients had to have two sets of normal resting vital signs (pulse, respiration rate, blood pressure and oxygen saturation) measured within a five-minute interval. The one-minute sit-stand test began with placing the patient on a chair without armrest, with the legs in a 90-degree bend. The patient was asked to sit and stand as many times as possible during one minute without supporting with the arms. The oxygen saturation was monitored by pulse oximetry during the test and continuously one minute after completion of the test. The one-minute sit-stand test was considered to indicate decompensation (i.e. positive test) if the oxygen saturation decreased $>4$ percentage points and/or if the patient could not perform more than 12 sit-stand repetitions in one minute. The test was terminated and also considered positive if saturation fell below $<90 \%$ or if the patient was unable to continue the test [10, $18,19]$.

Following completion of the sit-stand test, ambulance personnel telemedically consulted with a physician for patient triage. The decision on whether to admit the patient to hospital or not was made by the physician.

\section{Data collection}


We collected data for this quality assurance project on all patients with suspected COVID-19 triaged to an ambulance response by a GP in the Central Denmark Region during the study period. Patients were identified through the electronic prehospital patient record (PPJ). Patient related data (age, gender, COVIDsymptoms, comorbidities, vital parameters, prehospital and hospital treatment, COVID-19 status and final diagnosis) were collected by the primary investigator from PPJ and from electronic in-hospital patient records. For patients tested with the sit-stand test, the result of the test was entered into PPJ by ambulance personnel on-scene. Study data were collected and managed by the primary investigator using REDCap electronic data capture tools (REDCap, 10.0.10, Vanderbilt University).

\section{Outcome measurements}

\section{Guidline adherence}

The primary outcome for this part of the study was the proportion of patients suscpected of COVID-19 sitstand tested before being treated and released on-scene. Secondary outcomes were 1) the proportion of patients fulfilling criteria for sit-stand test examined with the test and 2) the proportion of patients not fulfilling criteria for sit-stand test examined with the test.

\section{Results of the sit-stand test and patient outcome}

The primary outcome for this part of the study was the proportion of patients treated with oxygen within 7 days among patients decompensating vs patients not decompensation during the test. Secondary outcomes 1) were the proportion of patients admitted to intensive care unit within 7 days in patients decompensating during the one-minute sit-stand test vs patients not decompensating, 2) the proportion of patients treated and released on-scene and the proportion of patients seen in the Emergency Department but not admitted to hospital, respectively, in patients decompensating during the sit-stand test vs patients not decompensating and 3 ) the proportion of patients treated and released on-scene who were subsequently admitted to hospital, treated with supplementary oxygen, and/or were admitted to an intensive care unit.

\section{Statistical analysis}

We lacked key knowledge on the patient group, size of the group, number of patients decompensating and number of these who would need oxygen supplement. Thereby, the study was exploratory and sample size calculation was not performed. Patients were included during the ongoing pandemic and duration of the study depended on the duration of the initial pandemic wave.

Binary data were analyzed by $\mathrm{Chi}^{2}$-tests and continuous data were examined for normal distribution and analyzed with students t-test. The level of significance was set at 0.05 . Analyzes were performed using Stata 15 (StataCorp LCC, College Station, TX, USA).

\section{Results}


Data was collected on 160 patients with suspected COVID-19 triaged to ambulance response by general practioner between 6th April 2020 and 6th July 2020. Four patients were excluded (two due to missing identification and two were included twice). Thus, data on 156 unique patients were included in the study. Figure 2 displays an overall overview of patients included in the study; the number of patients fulfilling the criteria for sit-stand test, and the number actually tested in each group.

\section{Guideline adherence}

In total, 70/156 (44.8\%) patients fulfilled the guideline criteria for the one-minute sit-stand test. Of these patients, $56 / 70(80 \%)$ had the one-minute sit-stand test performed. Of patients not fulfilling the guideline criteria for the one-minute sit-stand test (i.e. older than 70 years of age and/or risk factors for serious disease and/or abnormal vital parameters), 30/86 (35\%) had the sit-stand test performed. Thus 86/156 (55\%) patients had the sit-stand test performed, including the 30 patients having sit-stand tested outside the guideline. Baseline characteristics of patients both where the sit-stand test was performed and not performed are given in Table 1. Generally, patients tested were younger and had less comorbidities than patients not tested.

In total, 52 patients were treated and released on-scene and 50/52 (96.2 \%) were tested with the sit-stand test before being treated and released (primary outcome of guideline adherence part). Of patients treated and released on-scene, 13/52 (25\%) were either older than 70 years of age or had risk factors for serious disease. Of these, $12 / 13$ (92.3\%) were tested before being released. 
Table 1

Baseline characteristics of patients

\begin{tabular}{|c|c|c|}
\hline & $\begin{array}{l}\text { Test performed } \\
N=86\end{array}$ & $\begin{array}{l}\text { Test not performed } \\
N=70\end{array}$ \\
\hline Age, median (IQR) & $52(35-64)$ & $70(53-82)$ \\
\hline Male, n (\%) & $42(48.8)$ & $42(60.0)$ \\
\hline Risk factors, n (\%) & $1(1.2)$ & $1(1.4)$ \\
\hline Immunodeficiency & $\mathbf{0}(0)$ & $0(0)$ \\
\hline Severely increased blood pressure & $8(9.3)$ & $12(17.1)$ \\
\hline Coronary artery disease & $2(2.3)$ & $11(15.7)$ \\
\hline Cancer & $1(1.2)$ & $5(7.1)$ \\
\hline Diabetes mellites with complications & $1(1.2)$ & $1(1.4)$ \\
\hline Liver disease & $33.5)$ & $45.7)$ \\
\hline Severe obesity (BMI 35-40) & $1112.8)$ & $21(30.0)$ \\
\hline Chronic pulmonary disease & $0(0)$ & $4(5.7)$ \\
\hline Chronic kidney disease & $0(0)$ & $2(2.8)$ \\
\hline Impaired coughing conditions & $1(1.2)$ & $0(0)$ \\
\hline \multicolumn{3}{|l|}{ Pregnancy } \\
\hline Covid-19 symptoms, n (\%) & $48(48.8)$ & $31(44.2)$ \\
\hline Coughing & $20(23.3)$ & $30(42.9)$ \\
\hline Fever & $9(10.5)$ & $6(8.6)$ \\
\hline Myalgia & $6(7.0)$ & $9(12.9)$ \\
\hline Fatigue & $37(43.0)$ & $38(54.3)$ \\
\hline \multicolumn{3}{|l|}{ Dyspnea } \\
\hline Positive covid-19 test*, n (\%) & $6(7.0)$ & $4(5.7)$ \\
\hline
\end{tabular}

*Positive COVID-19 test within 14 days prior to and 30 days after inclusion Results of the sit-stand test and patient outcome

In total, 17/86 (19.7\%) of patients tested with the sit-stand test decompensated during the test. 9/17 $(52.9 \%)$ of patients who decompensated during the sit-stand test were treated with oxygen within 7 days vs $2 / 69(2.9 \%)$ of patients who did not decompensate during the test $(p<0.001)$ (Table 2$)$. This difference was only significant in the group of elderly, comorbid patients tested outside the guideline. In this group, 
$11 / 30(37 \%)$ decompensated during the test, and of these 8/11 (73\%) patients were treated with oxygen within 7 days compared to $0 / 19(0 \%)$ in the group of patients not decompensation $(p<0.001)$. In the group of patients tested inside the guideline (i.e. younger patients with no risk factors for serious disease), $6 / 56(11 \%)$ decompensated during the test, and of these patients $1 / 6(17 \%)$ were treated with oxygen within 7 days compared to $2 / 50(4 \%)$ in the group not decompensating $(p<0.193)$.

In total, $0 / 17(0 \%)$ of patients decompensating during the test were treated and released on-scene vs $50 / 69$ (72.5\%) of patients not decompensating (Table 2). Among patients treated and released on-scene, $7 / 69(10.1 \%)$ were admitted to hospital within 7 days. Two patients required supplementary oxygen and one patient was treated in the intensive care unit and mechanically ventilated - this patient had a positive COVID-19 test. Patient characteristics, admission cause, diagnosis and outcome measures for patients treated and released on-scene but admitted to hospital within 7 days are given in appendix 1 .

Table 2

Outcome measurements for patients with sit-stand test performed

\begin{tabular}{|c|c|c|}
\hline & $\begin{array}{l}\text { Decomp + } \\
\mathrm{N}=17\end{array}$ & $\begin{array}{l}\text { Decomp - } \\
\mathrm{N}=69\end{array}$ \\
\hline Triage & $13(76.5)$ & $12(17.4)$ \\
\hline Hospital admission, $\mathrm{n}(\%)$ & $4(23.5)$ & $7(10.1)$ \\
\hline ED but not admitted, $\mathrm{n}(\%)^{\star}$ & $0(0.0)$ & $50(72.5)$ \\
\hline \multicolumn{3}{|l|}{ Treated and released on-scene, $\mathrm{n}(\%)$} \\
\hline Length of stay, index admission (days), mean (min-max) & $3.5(1-16)$ & $1.8(1-7)$ \\
\hline Oxygen treatment & $9(52.9)$ & $0(0.0)$ \\
\hline During index admission, $\mathrm{n}(\%)$ & $0(0.0)$ & $2(2.9)$ \\
\hline \multicolumn{3}{|l|}{ Later, within 7 days, $\mathrm{n}(\%)^{\star \star}$} \\
\hline Intensive care unit admission, $\mathrm{n}(\%)$ & $0(0.0)$ & $\mathbf{0}(0.0)$ \\
\hline During index admission, $\mathrm{n}(\%)$ & $0(0.0)$ & $1(16.7)$ \\
\hline \multicolumn{3}{|l|}{ Later, within 30 days, $\mathrm{n}(\%) \star \star$} \\
\hline Later admission within 7 days, $\mathrm{n}(\%)^{\star \star}$ & $0(0.0)$ & $7(10.1)$ \\
\hline Death within 30 days, $n$ (\%) & $1(5.9)$ & $\mathbf{0}(0.0)$ \\
\hline Positive covid-19 test ${ }^{\star \star \star}, \mathrm{n}(\%)$ & $1(5.9)$ & $5(7.2)$ \\
\hline
\end{tabular}

Decomp +: patients who decompensated during the one-minute sit-stand test

Decomp -: patients who did not decompensate during the one-minute sit-stand test

*Seen in the emergency department, but not admitted to hospital 
**Hospital admissions later than an index admission

***Positive COVID-19 test within 14 days prior to and 30 days after inclusion

\section{Discussion}

This prospective cohort study investigated implementation and results of a prehospital one-minute sitstand test for identifying patients in risk of deterioration among seemingly stable patients with suspected COVID-19 infection triaged by telephone consult by a GP to an ambulance response. The study demonstrated that $96 \%$ of patients were tested before being treated and released on-scene. However, a large percentage of elderly patients with risk factors for serious disease were also tested outside the guideline using the sit-stand test. Twenty percent of patients decompensated during the test and of these patients, $53 \%$ were treated with supplementary oxygen within 7 days compared to $3 \%$ patients in the group of patients, who did not decompensate during the test. Interestingly, the difference was only significant in the group of elderly and/or comorbid patients tested outside the guideline.

Thus, in the present study, $35 \%$ of patients with risk factors for serious disease were tested in discrepancy with the guideline. It is well known that adherence to guidelines is not $100 \%$. A study by Juelsgaard et al. investigated to which extent the Danish Helicopter Emergency Medical Service staff adhere to the guideline for handling of patients suffering from traumatic brain injury (TBI). The adherence was $69 \%$ in a non-TBI group and $74 \%$ in a TBI-group of patients [20]. In order to increase adherence to guideline, Marino et al. implemented an intervention of training, video and decision tools and showed an increase from $29 \%$ pre-intervention to $74 \%$ post-intervention [21]. In the present study, both video material and decision tools were at all time available to the emergency medical personnel, but the rapid development of the ongoing pandemic may have limited the possibility for ongoing education and follow up.

Other studies have addressed the challenge of predicting outcome in COVID-19, but no clinical characteristics can isolated predict outcome [22]. Guo et al. retrospectively examined the ability of an early warning score including age, chronic diseases, neutrophil/leucocyte ratio, C-reactive protein and Ddimer to predict the clinical course of patients with COVID-19. By stratifying patients into low-, mediumand high risk groups they found significant difference in incidence of deterioration [23]. Prehospital use of this score would require extensive use of point-of-care diagnostics not presently available in most settings.

The absolute number of patients suffering COVID-19 in this study was very low, and thus the study gives no valid information about the predictive ability of the sit-stand test in patients with COVID-19. As such, the most interesting finding of this study is probably that the sit-stand test seems to predict detoriation risk in a group of elderly, comorbid patients in the prehospital setting. Although this study does suffer from selection bias in this population, the results warrants future studies on the value of the sit-stand test as a more general predictive tool for decision-making in this population that increases in size and complexity [24]. 
In the present study, 7/50 (14\%) patients treated and released on-scene were readmitted within 7 days. In a study by Raaber et al., also from the Central Denmark Region, the proportion of readmissions within 30 days after treat and release was $2.7 \%$ [16]. This difference may be caused by differences in patient population and triage (i.e. an overweight of 1-1-2 versus patients triaged by GP). A high focus on easing the burden on the health care system during the COVID-19 pandemic may also have aggravated this issue in the present study. The incidence of COVID-19 among patients included in this study was only $10 / 156$ (7\%). Thus, in a setting with a relative low COVID-19 disease transmission, rapid changes in the organization of the health care system in order to manage the pandemic may have caused inappropriate management of other patient groups.

\section{Strength And Limitations}

The pragmatic implementational approach that highly reflects daily clinical practice during the COVID-19 pandemic is a strength to the study. The one-minute sit-stand test was implemented as standard routine procedure in all ambulances in the Central Denmark Region in April 2020 with written instructions and video material through usual communication platforms to the ambulance personnel. The Danish Civil Registration System and PPJ ensure comprehensive data collection and allows the investigator to collect high-quality pre- and in-hospital information regarding patient treatment and outcome, which is considered an important strength.

The relatively vague definition of "suspected COVID-19" in a population that ended up with a low prevalence of COVID-19 makes the studied group of patients very heterogenous and the findings of the study are not generalizable to "suspected COVID-19" patients high-incidence parts of the world. This affects the external validity of the results. In addition, the sit-stand test was used outside the guideline also in a group of elderly comorbid patients - and most likely selectively in this group of patients. This may have introduced selection bias.

The primary end-point, supplemental oxygen treatment within 7 days, may have been underestimated in the group of patients not decompensating during the sit-stand test, as they were less likely to be admitted to hospital. This may have falsely increased the predictive potential of the sit-stand test.

\section{Conclusion}

The sit-stand test was implemented in $96 \%$ of patients treated and released on-scene, but was also used in elderly patients with risk factors for serious disease. Decompensation was observed in $20 \%$ of patients and was associated with oxygen treatment within 7 days - but only in the elderly group of patients with risk factors for serious disease. $10 \%$ of patients treated and released on-scene were admitted to hospital within 7 days. The prevalence of COVID-19 was low in this population, and data may be subject to bias, but the findings in especially the group of elderly comorbid patients generates the hypothesis that physical exercise testing may be usefull for decision making in emergency settings. 


\section{Declarations}

\section{Ethics}

The Central Denmark Region Committee on Biomedical and Research Ethics reviewed the study protocol and concluded that it was exempt from formal ethical approval and informed consent was waivered. Patients could at any time deny participation in the exercise test.

All collected data adhere to current legislation in the Danish Data Protection Act and performed in accordance with the Declaration of Helsinki. Patients were not exposed to additional invasive procedures or alteration of treatment. Participation in the study did not cause patient- nor system-delay. Participants did not receive any compensation for participation in the project.

All patients were attended by emergency medical technicians and/or paramedics staffing a fully equipped ambulance and the one-minute sit-stand test was implemented as a routine procedure. All ambulance personnel were capable of initiating oxygen therapy and providing basic life support in case of serious decompensation.

\section{Consent for publication}

Not applicable

\section{Availability of data and materials}

The dataset generated and analyzed during the current study is not public available due to sensitive personal data (civil registration number). Data is available from corresponding author upon reasonable request in anonymized form.

\section{Competing interests}

None of the authors has conflicts of interest.

\section{Funding}

The study is funded by the Grundfos s Foundation. The foundation had no influence on results or the publishing of data.

\section{Authors' contributions}

MB, JK, AB, UV and SH designed the study. JK and AB collected data. JK and MB performed datamanagement and data-analysis. JK and MB wrote the main manuscript text and prepared figures and tables. All authors reviewed the manuscript.

\section{Acknowledgments}


The authors thank Kenneth Studgaard Damholdt and Hans Christian Strikert for their participation in production and distribution of information material to relevant personnel. We thank all participating ambulance personnel in the Central Denmark Region.

\section{References}

1. Grasselli, G., A. Pesenti, and M. Cecconi, Critical Care Utilization for the COVID-19 Outbreak in Lombardy, Italy: Early Experience and Forecast During an Emergency Response. JAMA, 2020. 323(16): p. 1545-1546.

2. WHO. WHO Director-General's opening remarks at the media briefing on COVID-19-11 March 2020. 2020 [cited 2020; Available from: https://www.who.int/dg/speeches/detail/who-director-general-sopening-remarks-at-the-media-briefing-on-covid-19--11-march-2020.

3. Rothan, H.A. and S.N. Byrareddy, The epidemiology and pathogenesis of coronavirus disease (COVID19) outbreak. J Autoimmun, 2020: p. 102433.

4. Burdick, $\mathrm{H}$., et al., Prediction of respiratory decompensation in Covid-19 patients using machine learning: The READY trial. Comput Biol Med, 2020. 124: p. 103949.

5. Wu, Z. and J.M. McGoogan, Characteristics of and Important Lessons From the Coronavirus Disease 2019 (COVID-19) Outbreak in China: Summary of a Report of 72314 Cases From the Chinese Center for Disease Control and Prevention. Jama, 2020. 323(13): p. 1239-1242.

6. Orginazation, W.H. Clinical management of severe acute respiratory infection (SARI) when COVID-19 disease is suspected. 2020; Available from:

https://apps.who.int/iris/bitstream/handle/10665/331446/WHO-2019-nCoV-clinical-2020.4eng.pdf? sequence $=1$ \&isAllowed $=y$.

7. Sun, X., et al., Cytokine storm intervention in the early stages of COVID-19 pneumonia. Cytokine Growth Factor Rev, 2020. 53: p. 38-42.

8. Hu, B., S. Huang, and L. Yin, The cytokine storm and COVID-19. J Med Virol, 2020.

9. Crook, S., et al., A multicentre validation of the 1-min sit-to-stand test in patients with COPD. Eur Respir J, 2017. 49(3).

10. Gruet, M., et al., The 1-Minute Sit-to-Stand Test in Adults With Cystic Fibrosis: Correlations With Cardiopulmonary Exercise Test, 6-Minute Walk Test, and Quadriceps Strength. Respir Care, 2016. 61(12): p. 1620-1628.

11. Marius-Gabriel, S., et al., 1 Minute Sit-to-Stand Test versus 6 minutes walk test: methods of evaluating exercise capacity in COPD patients. European Respiratory Journal, 2017. 50(suppl 61): p. PA2496.

12. Briand, J., et al., The 1-minute sit-to-stand test to detect exercise-induced oxygen desaturation in patients with interstitial lung disease. Ther Adv Respir Dis, 2018. 12: p. 1753466618793028.

13. Rasekaba, T., et al., The six-minute walk test: a useful metric for the cardiopulmonary patient. Intern Med J, 2009. 39(8): p. 495-501. 
14. Statistik, D. Area and population - Central Denmark Region. 2020 06.11.2020].

15. Andersen, M.S., et al., Implementing a nationwide criteria-based emergency medical dispatch system: a register-based follow-up study. Scand J Trauma Resusc Emerg Med, 2013. 21: p. 53.

16. Raaber, N., et al., Telemedicine-based physician consultation results in more patients treated and released by ambulance personnel. Eur J Emerg Med, 2018. 25(2): p. 120-127.

17. Prehospital Emergency Medical Services, C.D.R. COVID-19. Afs/utning af COVID-19 patienter visiteret af egen læge. 2020 [cited 2020; Available from: https://edok.rm.dk/edok/admin/GUl.nsf/Desktop.html?Open\&login.

18. ATS statement: guidelines for the six-minute walk test. Am J Respir Crit Care Med, 2002. 166(1): p. 111-7.

19. Lama, V.N., et al., Prognostic value of desaturation during a 6-minute walk test in idiopathic interstitial pneumonia. Am J Respir Crit Care Med, 2003. 168(9): p. 1084-90.

20. Juelsgaard, J., et al., Prehospital treatment of patients with acute intracranial pathology: adherence to guidelines and blood pressure recommendations by the Danish Air Ambulance. Scand J Trauma Resusc Emerg Med, 2018. 26(1): p. 68.

21. Marino, M.C., et al., Improving Prehospital Protocol Adherence Using Bundled Educational Interventions. Prehosp Emerg Care, 2018. 22(3): p. 361-369.

22. Wynants, L., et al., Prediction models for diagnosis and prognosis of covid-19 infection: systematic review and critical appraisal. Bmj, 2020. 369: p. m1328.

23. Yabing Guo, Y.L., Jiatao Lu, Rong Fan, Fuchun Zhang, Xueru Yin, Zhihong Liu, Qinglang Zeng, Jing Yuan, Shufang Hu, Qiongya Wang, Baolin Liao, Mingxing Huang, Sichun Yin, Xilin Zhang, Rui Xin, Zhanzhou Lin, Changzheng Hu, Boliang Zhao, Ridong He, Minfeng Liang, Zheng Zhang, Li Liu, Jian Sun, Lu Tang, Lisi Deng, Jinyu Xia, Xiaoping Tang, Lei Liu, Jinlin Hou. Development and validation of an early warning score (EWAS) for predicting clinical deterioration in patients with coronavirus disease 2019. 2020 [cited 2020 16/11-20]; Available from: https://www.medrxiv.org/content/10.1101/2020.04.17.20064691v1.

24. Christensen, E.F., et al., Trends in diagnostic patterns and mortality in emergency ambulance service patients in 2007-2014: a population-based cohort study from the North Denmark Region. BMJ Open, 2017. 7(8): p. e014508.

\section{Figures}




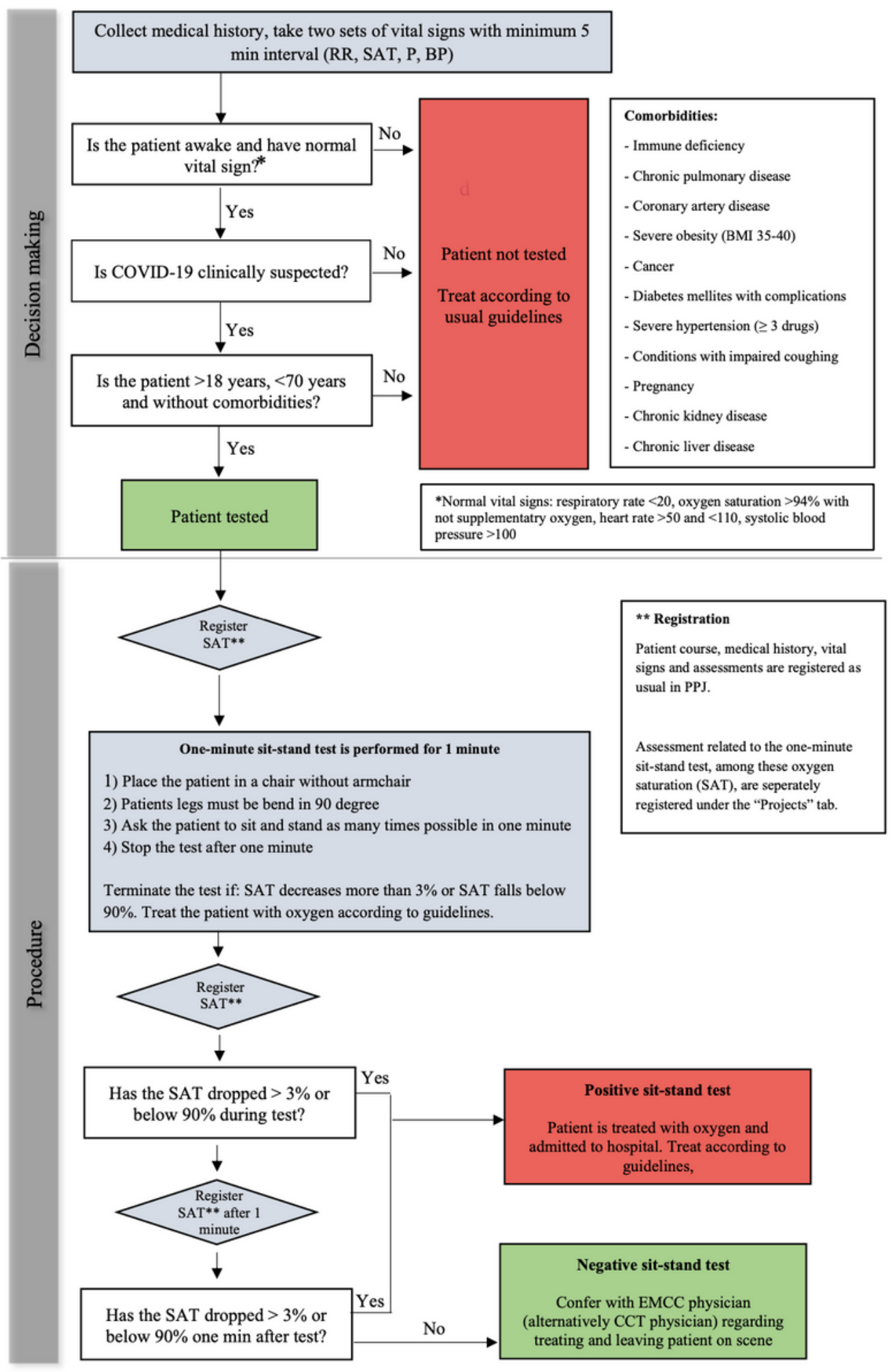

\section{Figure 1}

Sit-stand-test decision-making flow-chart (English translation) 


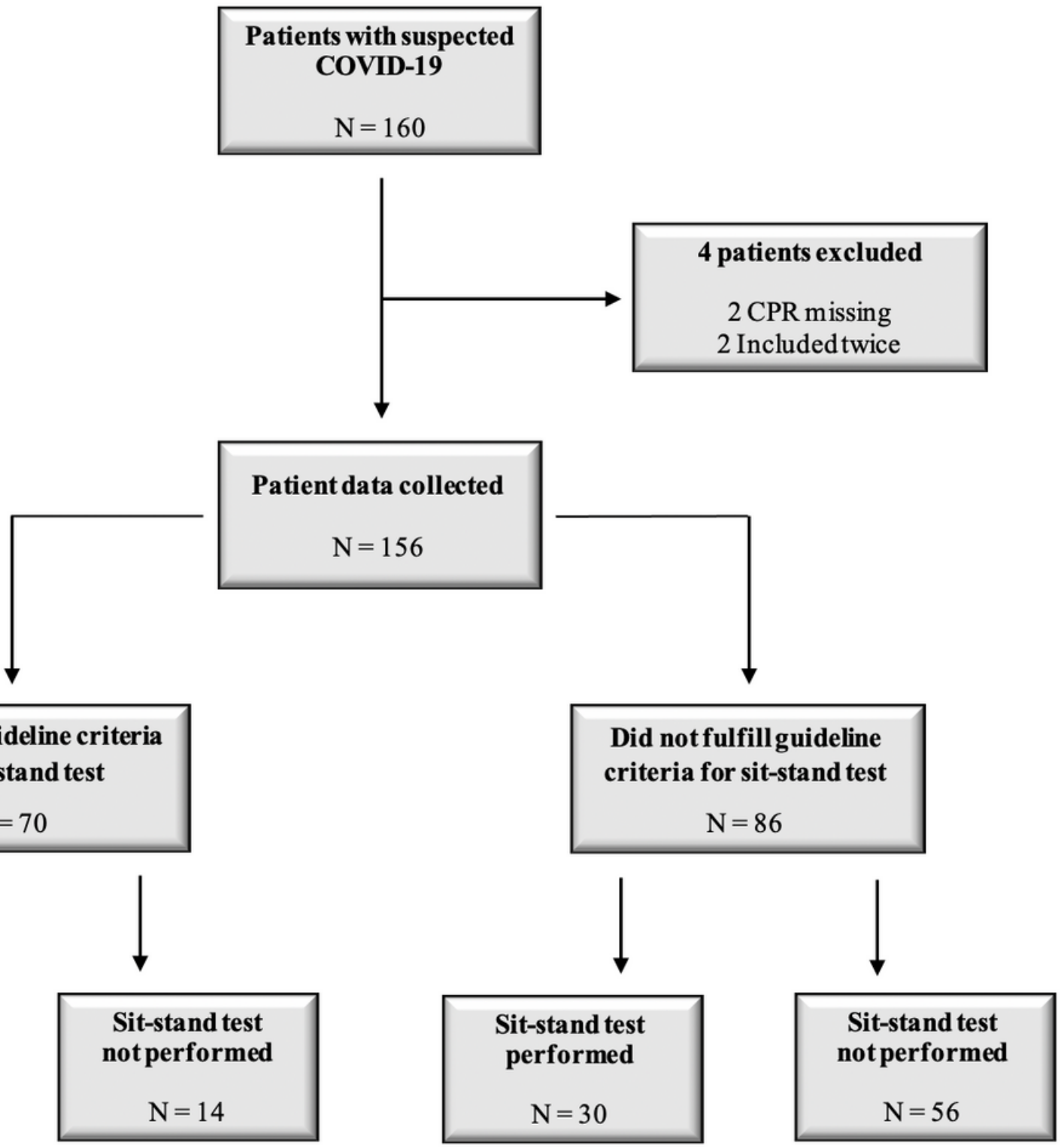

Figure 2

Flowchart of guideline implementation for the sit-stand test

\section{Supplementary Files}

This is a list of supplementary files associated with this preprint. Click to download.

- supplementarysitstandtestsubmissionBMC16.09.21.docx 